\title{
Evaluating COVID-19 booster vaccination strategies in a partially vaccinated population: a modeling study.
}

Clément R Massonnaud, MD, ${ }^{1,2,{ }^{\star}}$ Jonathan Roux, $\mathrm{PhD},{ }^{1,{ }^{*}}$ Vittoria Colizza, $\mathrm{PhD},{ }^{3}$ Pascal Crépey, $\mathrm{PhD}^{1}$

${ }^{*}$ Authors contributed equally to the work.

Affiliations:

1: Univ Rennes, EHESP, REPERES « Recherche en Pharmaco-Epidémiologie et Recours aux Soins $\gg-$ EA 7449, 35043 Rennes, France

2: Biostatistics Unit, University hospital Charles Nicolle, Rouen, Normandie, France.

3: INSERM, Sorbonne Université, Pierre Louis Institute of Epidemiology and Public Health, Paris, France

Corresponding author:

Pascal Crépey

pascal.crepey@ehesp.fr 


\section{Abstract}

Background. As evidence shows that vaccine immunity to COVID-19 wanes with time and decreases due to variants, several countries are implementing booster vaccination campaigns. The objective of this study was to analyze the morbidity and mortality burdens of different primary and booster vaccination strategies against COVID-19, using France as a case study.

Methods. We used a deterministic, age-structured, compartmental model fitted to hospital admission data and validated against sero-prevalence data in France to analyze the impact of primary and booster vaccination strategies on morbidity and mortality assuming waning of immunity and increased virus transmissibility during winter.

Findings. Strategies prioritizing primary vaccinations were systematically more effective than strategies prioritizing boosters. Regarding booster strategies targeting different age groups, their effectiveness varied with the levels of virus transmissibility, and according to the assumed loss of immunity for each age group. If the immunity reduction affects all age groups, people aged 30 to 49 years should be boosted in priority, even for low transmissibility levels. If the immunity reduction is restricted to people older than 65 years, boosting younger people becomes effective only above certain levels of transmissibility.

Interpretation. Increasing the primary vaccination coverage should remain a priority to reduce morbidity and mortality due to COVID-19. If a plateau of primary vaccination has been reached, boosting immunity in younger age-groups could prevent more hospitalizations and deaths than boosting the immunity of older people, especially under conditions increasing SARS-CoV-2 transmissibility, or when facing new variants.

Funding. The study was partially funded by the French national research agency through project SPHINX-17-CE36-0008-0. 


\section{Research in context}

\section{Evidence before this study}

Many countries have started booster vaccination programs against Covid-19, while others are still struggling to vaccinate their population. However, evidence is scarce regarding the optimal vaccination strategy to pursue in a rapidly evolving epidemiological context. A search of the literature on Nov 27 2021, using the terms (booster OR third dose) AND vaccine AND strategy AND (COVID* OR SARS*) AND (effect OR impact), returned 45 studies on PubMed and 1602 on medRxiv. However, very few studies assessed the public health impact of a booster strategy, and none of them compared different allocations strategies between primary and booster vaccinations, or investigated which age-group should be targeted for booster vaccination to maximize the public health impact of the strategy.

\section{Added value of this study}

Using an epidemiological model able to replicate the dynamic of the SARS-CoV-2 epidemic and able to account for the use of multiple vaccines and booster, we analyzed the effectiveness of different vaccination strategies, either based on prioritization of primary vaccination versus booster, or based on the age-group targeted for the booster vaccination. We evaluated the strategies in terms of hospitalizations and deaths avoided, in various epidemic scenarios during winter 2021-2022. To our knowledge, this is the first modeling study evaluating such strategies. We found that increasing primary vaccination of all adults is always more beneficial than giving a booster dose to elderly individuals, and that the age-group to target for a booster dose for optimal effectiveness depends on the level of transmission of the virus. As the level of SARSCoV-2 transmission increases, boosting immunity in younger age-groups becomes the most effective strategy to decrease hospitalizations and deaths in the general population.

\section{Implications of all available evidence}

Countries that have not reached the plateau of primary vaccination should focus their effort towards extending the overall primary vaccination coverage rather than boosting the immunity of fully vaccinated people, even for elderly individuals that may be facing waning immunity. When considering booster vaccination, the choice of which age groups to target should consider the level of virus transmissibility in the population. Considering the emergence of new, more transmissible SARS-CoV-2 variants, increasing the worldwide vaccination coverage should remain a priority. 


\section{Introduction}

Several highly effective COVID-19 vaccines have been made available worldwide since the end of $2020 .^{1-7}$ In France, four vaccines have been authorized: Comirnaty ${ }^{\circledR}$ (Pfizer/BioNTech), Spikevax ${ }^{\circledR}$ (Moderna), Vaxzevria ${ }^{\circledR}$ (AstraZeneca), and COVID-19 Vaccine Janssen (Janssen-Cilag) ${ }^{8}$ and used in vaccination campaigns to help mitigate the epidemic. The vaccination campaign started with the elderly population and at-risk population in January 2021, and was progressively extended to younger age-groups down to 12 years of age. The vaccination coverage for people aged 65 years or more reached $60 \%$ in June 2021, and plateaued at the end of the summer at $85 \%$. However, coverage remained low in specific senior age classes; for example, only $78 \%$ of $80+$ received a full vaccination. The vaccination of individuals aged 18 to 64 started later on during the spring, and really took off in May for the 50-64 age groups, and June for the 18-49 age groups. The vaccination coverage increased rapidly but then slowed down significantly by the end of the summer, at levels ranging from 70\% (30-39 age group) to $85 \%$ (60-64 age group). The vaccination of the 12-17 age group started in July, and reached 50\% at the end of August. ${ }^{9}$

Among the SARS-CoV-2 variants that spread worldwide, the variant of concern Alpha (VOC B.1.1.7) became dominant in metropolitan France in February 2021, ${ }^{10}$ and was then replaced by the more transmissible Delta VOC (B.1.617.2), which has been dominant since July $2021 .^{11}$ Several studies now suggest that the efficacy of vaccines against the Delta VOC is lower than against other variants. ${ }^{4-6,12}$ Moreover, recent studies show that vaccine immunity, especially regarding infection and symptomatic form, is waning with time. ${ }^{12-14}$ Therefore, many countries have recommended vaccine booster shots for all or part of their already vaccinated population. In France, only people aged 65 years or more (and specific people at risk) were initially eligible to receive a booster dose, administered at least 6 months after completion of the primary vaccination. Recommendations have recently extended to the $18+$ vaccinated population, similarly to other countries. ${ }^{15}$

With the surging wave in Europe, and in the context of global vaccine supply constraints, it is important to assess the effectiveness of booster vaccination campaigns. The objective of this study was to analyze the impact, on hospital admissions and deaths, of COVID-19 primary and booster vaccination strategies targeting different age groups, under various hypotheses of vaccine efficacy and virus transmission levels, using France as a case study.

\section{Methods}

\section{COVID-19 transmission model}

We used a deterministic, age-structured, compartmental epidemic model, based on demographic and age profile of the population of metropolitan France. It is described in supplementary material and described in previous publications. ${ }^{16}$ This model accounted for differences in susceptibility, severity and contacts across age groups. ${ }^{17-19}$ To consider the impact of vaccines, we updated the model to a multi-branch transmission model, duplicating the main branch for each vaccine considered (Supplementary Figure S2). In this framework, 
non-vaccinated individuals from susceptible and removed compartments could be vaccinated and integrate a vaccinated branch. This multi-branch model allowed us to use vaccine specific efficacies in each branch, and therefore to model booster doses. The main outcomes returned by the model were the daily number of newly exposed and hospitalized individuals, among others.

\section{Parametrization}

The model is fitted dynamically on the number of observed hospitalizations from the start of the pandemic in March 2020 to September 2021 (supplementary materials). We use a 7-day sliding window to estimate transmission parameters and shift the window by steps of 3 days. This method allows us to precisely reproduce epidemiological dynamics due to variations of transmissibility related to external factors like mitigation measures or season. To account for the impact of VOCs on transmission parameters, we considered a $40 \%$-increase in the risk of hospitalization between Alpha VOC and wild type ${ }^{20}$ and a $80 \%$-increase between Delta VOC and Alpha VOC. ${ }^{4}$ Moreover, to take into account an increase of transmissibility of the virus due to seasonality during the simulated period, we progressively increased the transmission over two weeks, starting on September 20, 2021, and then held it constant until the end of the simulation (March 1st 2022), to reproduce a $41 \%$ increase in winter time ${ }^{21}$ of the average reproduction number estimated in August $\left(R_{\text {eff }}=1.0\right)$. In addition, we explored a range from $0 \%$ to $200 \%$ of increase of transmissibility due to seasonality. The model successfully reproduced the observed evolution of the epidemic in France, and estimates of the immune status of the population (Figure S14) are in accordance with what is reported by observational studies. ${ }^{22}$ Details about the model and its parameterization are presented in supplementary materials.

\section{Vaccine efficacies}

Our transmission model allowed us to consider vaccine efficacies against three COVID-19 stages: infection, symptomatic case, and hospitalization. We considered that infected who were vaccinated had the same risk of transmission as non-vaccinated. We chose to group vaccines in two categories: mRNA vaccines (Comirnaty ${ }^{\circledR}$ and Spikevax ${ }^{\circledR}$ ) and vector vaccines (Vaxzevria ${ }^{\circledR}$ and Janssen COVID-19 vaccine) due to the similarities in their characteristics and efficacies. We considered distinct efficacies according to the predominant VOC (Alpha or Delta) on the French territory and we only used efficacies after a complete vaccine scheme (2 doses for all vaccines except Janssen COVID-19 vaccine which had one dose). Given the complexity of modeling the differential waning of efficacy against infection, symptomatic case and hospitalization, we used a simplified approach where each efficacy is reduced at fixed time points and for specific agegroups. Two scenarios were considered when the Delta VOC was predominant: 1) a decrease in vaccine effectiveness only in 65 years and older, which would correspond to a case where this population was vaccinated first and is facing a waning of immunity, and 2) a decrease in all age groups, which would correspond either to a scenario where all age groups started the primary vaccination at the same time, and are facing a waning of immunity, or to a scenario where the immunity reduction is due to the Delta VOC itself, even for people recently vaccinated. Table 1 shows the values for the different vaccine efficacies conditioned to the predominant variant and the scenario that were used in the simulations. ${ }^{12}$ We assumed the booster dose confers an 
medRxiv preprint doi: https://doi.org/10.1101/2021.12.01.21267122; this version posted December 2, 2021. The copyright holder for this preprint (which was not certified by peer review) is the author/funder, who has granted medRxiv a license to display the preprint in perpetuity.

It is made available under a CC-BY-NC-ND 4.0 International license .

immunity against the Delta VOC equivalent to the one observed against the Alpha VOC which may be a conservative estimate compared to latest evaluations. ${ }^{23}$

Table 1 Retained efficacies against infection, symptomatic case and hospitalization for mRNA (Comirnaty ${ }^{\circledR}$ and Spikevax ${ }^{\circledR}$ ) and vector vaccines (Vaxzevria ${ }^{\circledR}$ and Janssen COVID-19 vaccine) according to the predominant SARS-CoV-2 variant

\begin{tabular}{|c|c|c|c|c|c|}
\hline & & Vaccine & Infection & $\begin{array}{c}\text { Symptomatic } \\
\text { case }\end{array}$ & $\begin{array}{c}\text { Hospitalizatio } \\
\mathbf{n}\end{array}$ \\
\hline \multirow{2}{*}{$\begin{array}{c}\text { Efficacy } \\
\text { against Alpha } \\
\text { VOC }\end{array}$} & \multirow[t]{2}{*}{ Base efficacy } & Vector vaccine & $73 \%{ }^{4}$ & $75 \%{ }^{5}$ & $86 \%{ }^{6}$ \\
\hline & & mRNA & $92 \%{ }^{4}$ & $94 \%{ }^{5}$ & $97 \%^{7}$ \\
\hline \multirow{5}{*}{$\begin{array}{c}\text { Efficacy } \\
\text { against Delta } \\
\text { VOC }\end{array}$} & \multirow[t]{2}{*}{ Base efficacy } & Vector vaccine & $60 \%{ }^{4}$ & $67 \% \%^{5}$ & $92 \%{ }^{6}$ \\
\hline & & mRNA & $79 \%{ }^{4}$ & $88 \%{ }^{5}$ & $96 \%{ }^{6}$ \\
\hline & \multirow[t]{2}{*}{$\begin{array}{l}\text { Decreased } \\
\text { efficacy }\end{array}$} & Vector vaccine & $53 \%^{12}$ & $53 \%^{12}$ & $92 \%{ }^{*}$ \\
\hline & & mRNA & $53 \%^{12}$ & $53 \%^{12}$ & $93 \%{ }^{12}$ \\
\hline & $\begin{array}{l}\text { Efficacy after } \\
\text { booster dose }\end{array}$ & mRNA & $92 \%$ & $94 \%$ & $97 \%$ \\
\hline
\end{tabular}

*We assumed that the waning of vector vaccine efficacy was similar to the one of mRNA vaccines, hence no waning on efficacy against hospitalization.

\section{Vaccination strategies}

We assessed the impact of different vaccination strategies starting on September 1st, 2021. Two distinct analyses are presented: (A) a comparison of vaccination strategies prioritizing primary vaccinations or booster shots in various proportions, and (B) a comparison of booster strategies targeting different age groups, in a context of plateauing primary vaccination. In both analyses, the effectiveness of each strategy is evaluated by the proportion of hospitalizations and deaths avoided, compared to an artificial reference scenario in which all vaccination (booster or primary) is stopped on September 1st, 2021.

In the first analysis, the strategies differed in the prioritization of 200,000 daily available doses: they could be allocated either as first or second doses for primary vaccinations, or as booster doses for people already fully vaccinated. For both primary vaccinations and booster doses, we 
assumed a maximum vaccination coverage of $90 \%$ of the eligible population (note that this level had already been reached by September 1st for the primary vaccination of people aged 70 to 79). Once this plateau was reached for one of the targets (primary vaccinations or booster doses), the remaining doses were all allocated to the other one (supplementary figure S4). The eligible population for primary vaccinations were individuals aged 12 years and older, and the people eligible to booster doses were individuals aged 65 years and older, in accordance with French recommendations. ${ }^{24}$ We evaluated five strategies ranging from all available doses allocated to new vaccinations, to all available doses allocated to booster vaccination, by steps of $50 \mathrm{k}$ doses from one allocation to another. To assess the impact on the results of the rate of vaccination, we performed sensitivity analyses considering a lower vaccination rate of 100,000 doses per day, and a higher vaccination rate of 400,000 doses per day.

In the second analysis, we simulated the administration of 10 million booster doses across five age groups: 30-49 years, 50-64 years, 30 years or older, 50 years or older, and 65 years or older, starting September 1st, 2021, at a rate of 200,000 doses per day. Primary vaccination was stopped on September 1st. We then analyzed the impact of different levels of increase of virus transmissibility due to seasonality from no increase to a $200 \%$-increase compared to the mean transmissibility level estimated during summer $2021\left(R_{\text {eff }}=1.0\right)$.

The model was run from March 2020 to the end of August 2021, calibrated to fit the number of daily hospitalizations observed in France over this period. Then, the different strategies were simulated from September 1st, 2021, to March 1st, 2022. We finally estimated the number of hospitalizations and deaths avoided in each vaccination or boosting strategy compared to the baseline scenario with no vaccination. All the analyses were performed using the $R$ software (version 4.1.1). The code of the model, as well as all the code producing the results, is available upon request.

\section{Role of funding source}

The funding source had no role in the study. 


\section{Results}

\section{Primary vaccination versus booster doses}

Figure 1 shows the effectiveness of vaccination strategies varying in the allocation of 200,000 daily doses between primary vaccination and booster doses compared to the baseline scenario in which all vaccination is stopped on September 1st, 2021. Strategies prioritizing primary vaccination were systematically more effective than strategies prioritizing boosters. If vaccine efficacy is decreased for all age groups, the increase in transmission during winter would lead to a wave of new hospitalizations, which would have been mitigated by any vaccination strategy (Figure 1-B). The strategy allocating all doses in priority to primary vaccinations would have been the most effective, resulting in a $62 \%$ reduction in hospitalizations over the period, whereas the strategy allocating all doses in priority to booster shots would have resulted in a $47 \%$ reduction (Figure 1-D); the other strategies having intermediate effectiveness. The analysis stratified by age group showed the same ranking of the strategies, even for 65 years and older, albeit with smaller differences (Figure 1-F). Even when the protection conferred by vaccines is decreased only for people aged 65 years or older, strategies prioritizing the boosting of this population were the least effective to prevent hospitalizations (Figures 1-A, 1-C, 1-E). The rankings of the strategies were identical regarding the number of deaths, and in the sensitivity analyses considering lower (100,000 doses per day) or higher (400,000 doses per day) vaccination rates (supplementary material). 
A
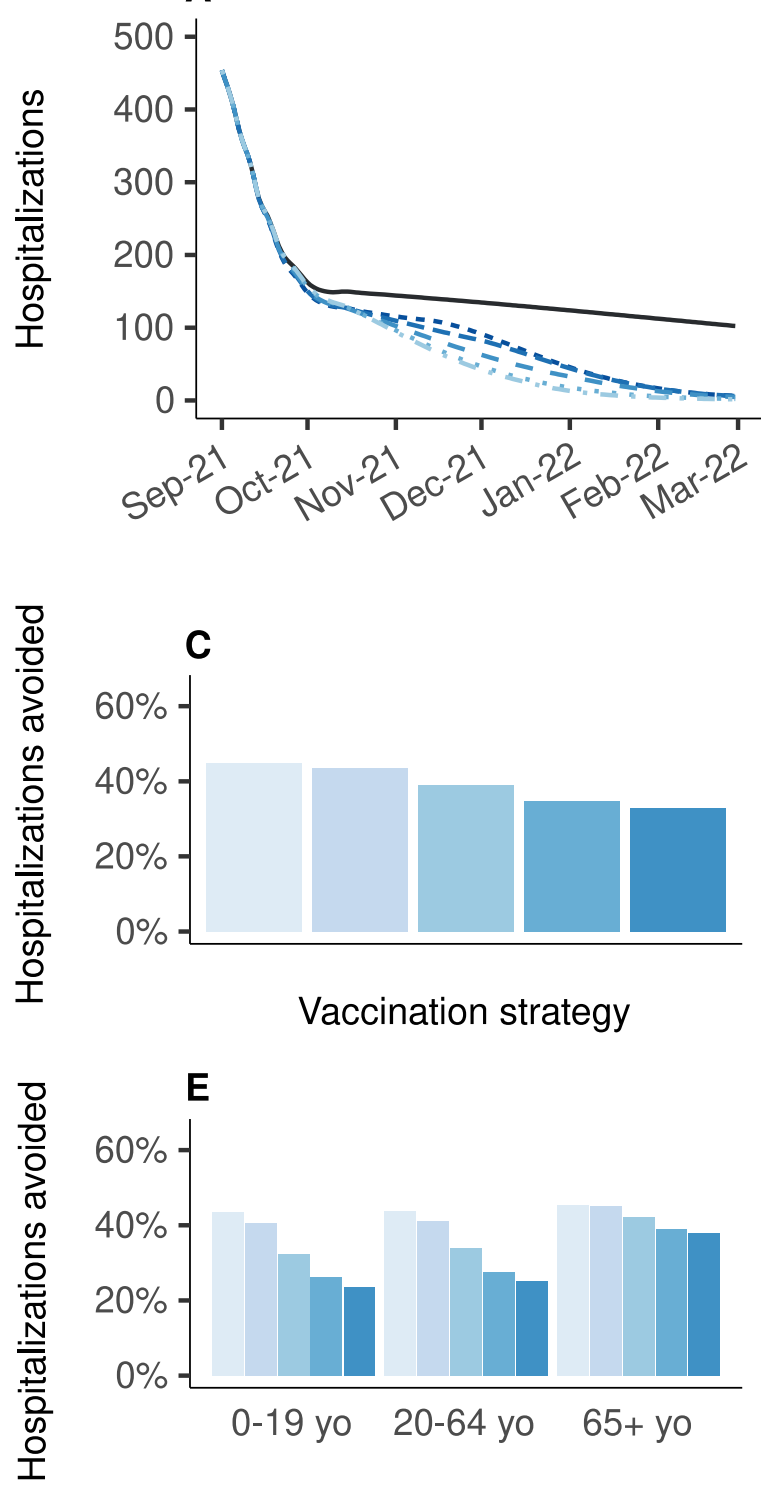

B

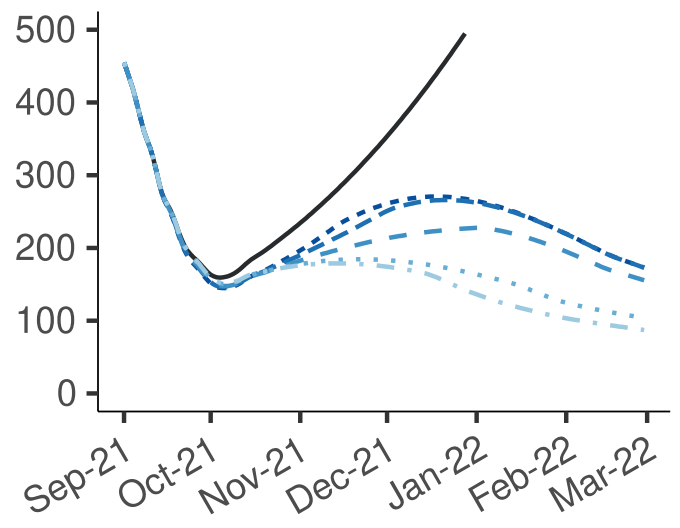

D

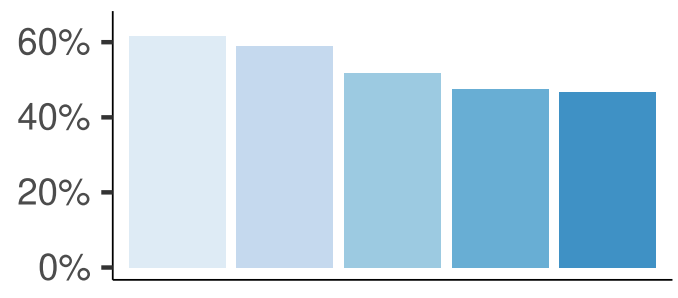

Vaccination strategy

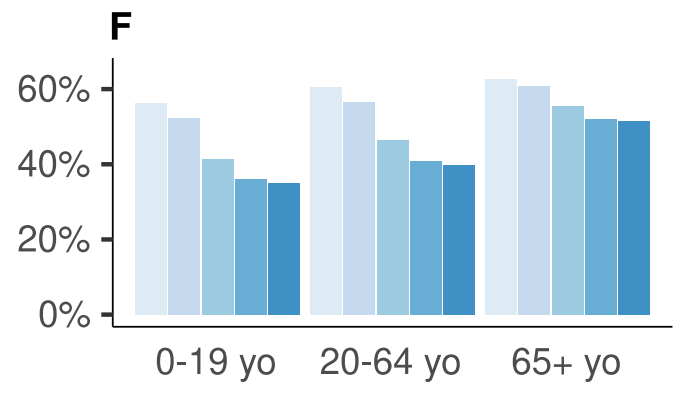

- No new vaccination, no booster

-.. Priority boosters $(0 \mathrm{k} / 200 \mathrm{k})$

-- Priority boosters $(50 \mathrm{k} / 150 \mathrm{k})$

- Priority equal (100k/100k)

-. Priority new vaccinations $(150 \mathrm{k} / 50 \mathrm{k})$

- Priority new vaccinations $(200 \mathrm{k} / 0 \mathrm{k})$

Figure 1. Effectiveness on hospitalizations of vaccination strategies varying in the allocation of 200,000 daily doses between primary vaccination and booster shots, over the period from September 1st, 2021 to March 1st, 2022, compared to a baseline scenario in which all vaccination is stopped on September 1st, 2021. (A, C, E) Vaccine efficacy decreased only for people aged 65 years and older. (B, D, F) Vaccine efficacy decreased for all age groups. (A,B) Daily new hospitalizations, (C, D) proportion of hospitalizations avoided, (E, F) proportion of hospitalizations avoided by age group. A prioritization strategy of $(150 \mathrm{k} / 50 \mathrm{k})$ means $150 \mathrm{k}$ daily doses for primary vaccination and $50 \mathrm{k}$ daily booster doses until $90 \%$ coverage of one target population is reached, then all $200 \mathrm{k}$ daily doses are allocated to the other target population. 


\section{Prioritization of booster doses}

Figure 2 shows the impact on the hospital admissions due to COVID-19 of 10 million booster shots depending on the targeted age groups, over the period from September 1st, 2021 to March 1st 2022, compared to a baseline scenario of no booster shots and no primary vaccination. If vaccine efficacy is decreased for all age groups (Figures 2-B, 2-D, 2-F), boosting people aged 30 to 49 years would be the most effective strategy, reducing the total number of hospitalizations by $71 \%$, while boosting people aged 65 years or older would lead to a $18 \%$ reduction only (Figure 2-D). In the other scenario, in which the efficacy is decreased only for people aged 65 years or older, the strategy targeting the 30-49 age group was the most effective, with $25 \%$ reduction. The strategy targeting the $30+$, and the one targeting the $65^{+}$, were next with similar levels of reduction in the total number of hospitalizations: $18 \%$ and $16 \%$, respectively (Figure 2-C). Figure 3 shows the same analyses regarding the deaths due to COVID19. If vaccine efficacy is decreased for all age groups, boosting people aged 30 to 49 years would still be the most effective strategy (Figure 3-D). However, if vaccine efficacy is decreased only for people aged 65 years or more, a boosting strategy targeting this population would be the most effective at reducing the total number of deaths (Figure 3-C). 

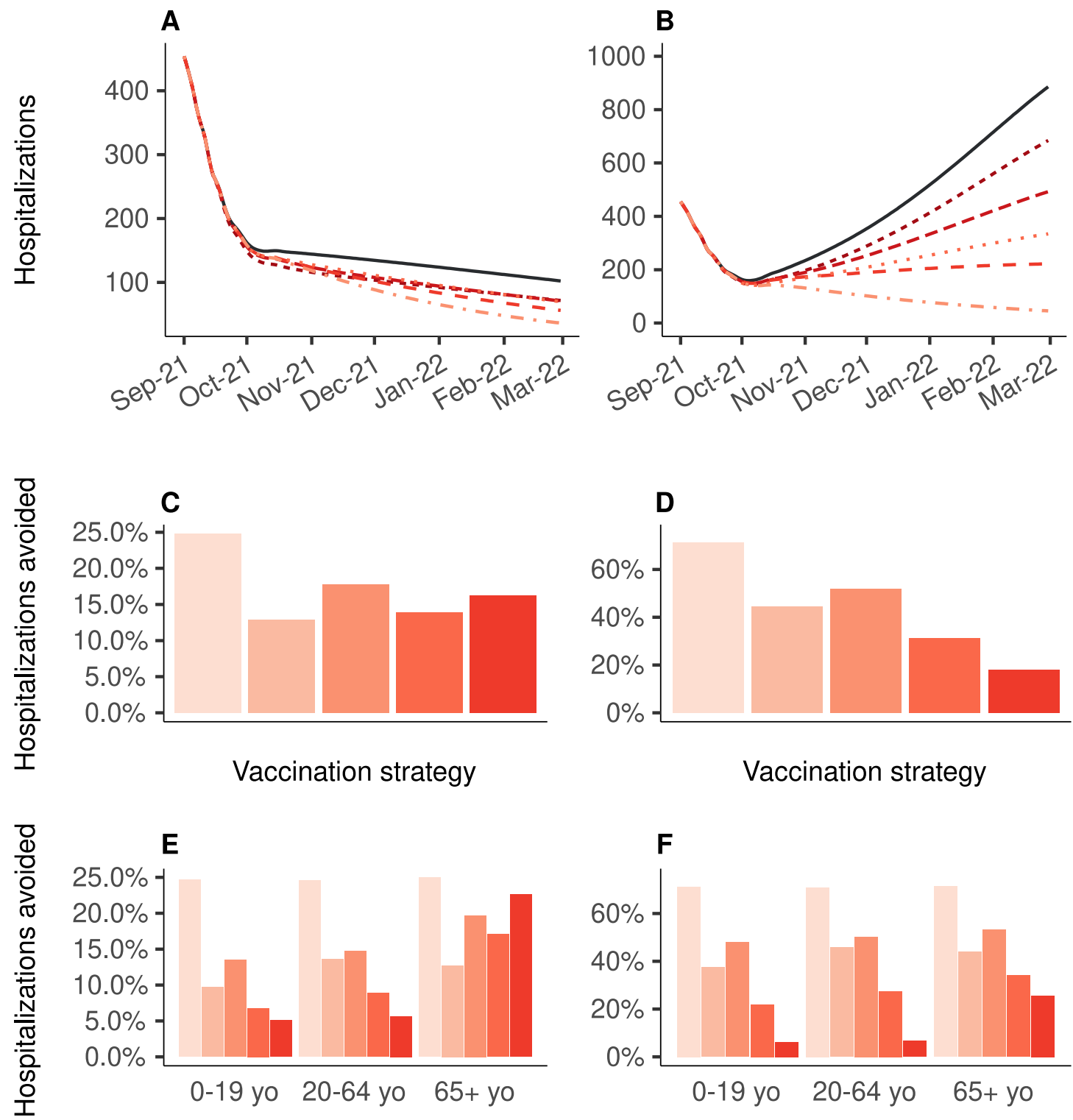

Figure 2. Impact on the hospital admissions of 10 million booster shots depending on the targeted age groups, over the period from September 1st, 2021 to March 1st, 2022, compared to a baseline scenario of no booster shots. In all strategies, primary vaccination is stopped on September 1st, 2021. (A, C, E) Vaccine efficacy decreased only for people aged 65 years and older. (B, D, F) Vaccine efficacy decreased for all age groups. (A, B) Daily new hospitalizations, (C, D) proportion of hospitalizations avoided, (E, F) proportion of hospitalizations avoided by age group. 

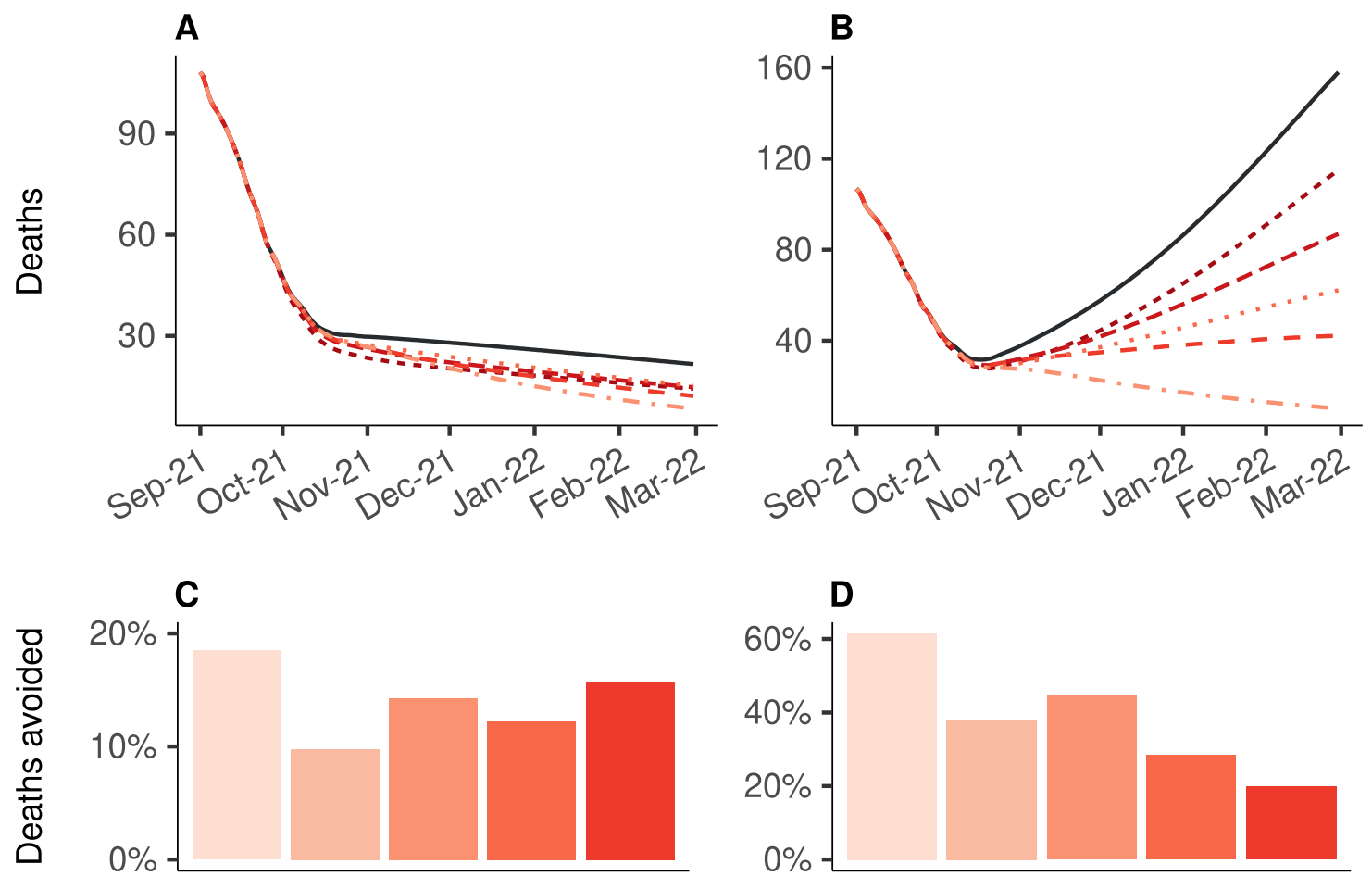

Vaccination strategy

\section{Vaccination strategy}
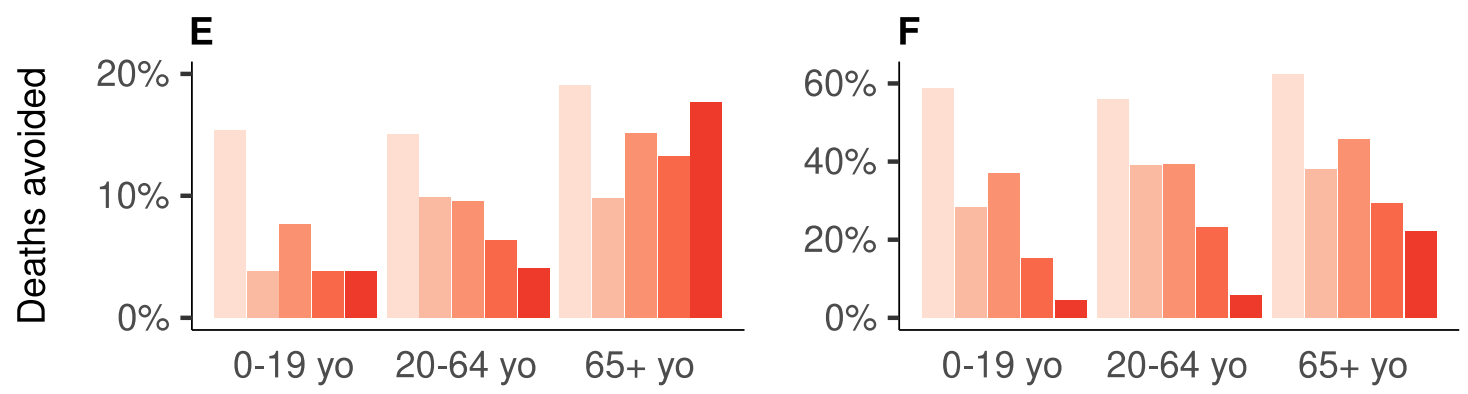

Figure 3. Impact on the number of deaths of 10 million booster shots depending on the targeted age groups, over the period from September 1st, 2021 to March 1st, 2022, compared to a baseline scenario of no booster shots. In all strategies, primary vaccination is stopped on September 1st, 2021. (A, C, E) Vaccine efficacy decreased only for people aged 65 years and older. (B, D, F) Vaccine efficacy decreased for all age groups. (A, B) Daily deaths, (C, D) proportion of deaths avoided, (E, F) proportion of deaths avoided by age group. Note that only the booster strategy targeting the 30-49yo had an impact on the percentage of deaths avoided in the 0 to 19yo on inset $\mathrm{E}$ (one death avoided). 


\section{Impact of variations of transmissibility}

Previous results were obtained considering a $41 \%$ increase of viral transmissibility during winter compared to the summer. As uncertainty still exists around the seasonality of SARS-CoV2 transmission, we also evaluated whether the prioritization of vaccination strategies is sensitive to variations of transmissibility, either due to seasonality or loss of efficacy of contact reduction measures. When vaccine efficacy was decreased for all age groups, the ranking of the strategies did not change with changes in transmissibility, and targeting the 30-49 age group remained the most effective boosting strategy to reduce both hospital admissions and deaths (Figures 4-B and 4-D). However, when the vaccine efficacy was decreased only for people aged 65 years or older, the ranking of the strategies varied depending on the assumed winter viral transmissibility (Figures 4-A and 4-C). For a 30\% transmissibility increase or more, relative to the mean transmissibility in summer (corresponding to $R_{\text {eff }}=1$ ), targeting the 30-49 age group was the most effective boosting strategy to reduce hospitalizations. Regarding the proportion of deaths avoided, the differences between the strategies were smaller, and a larger degree of transmissibility (>80\% increase) was needed to identify the $30-49$ age group as the optimal group to target with boosters. 

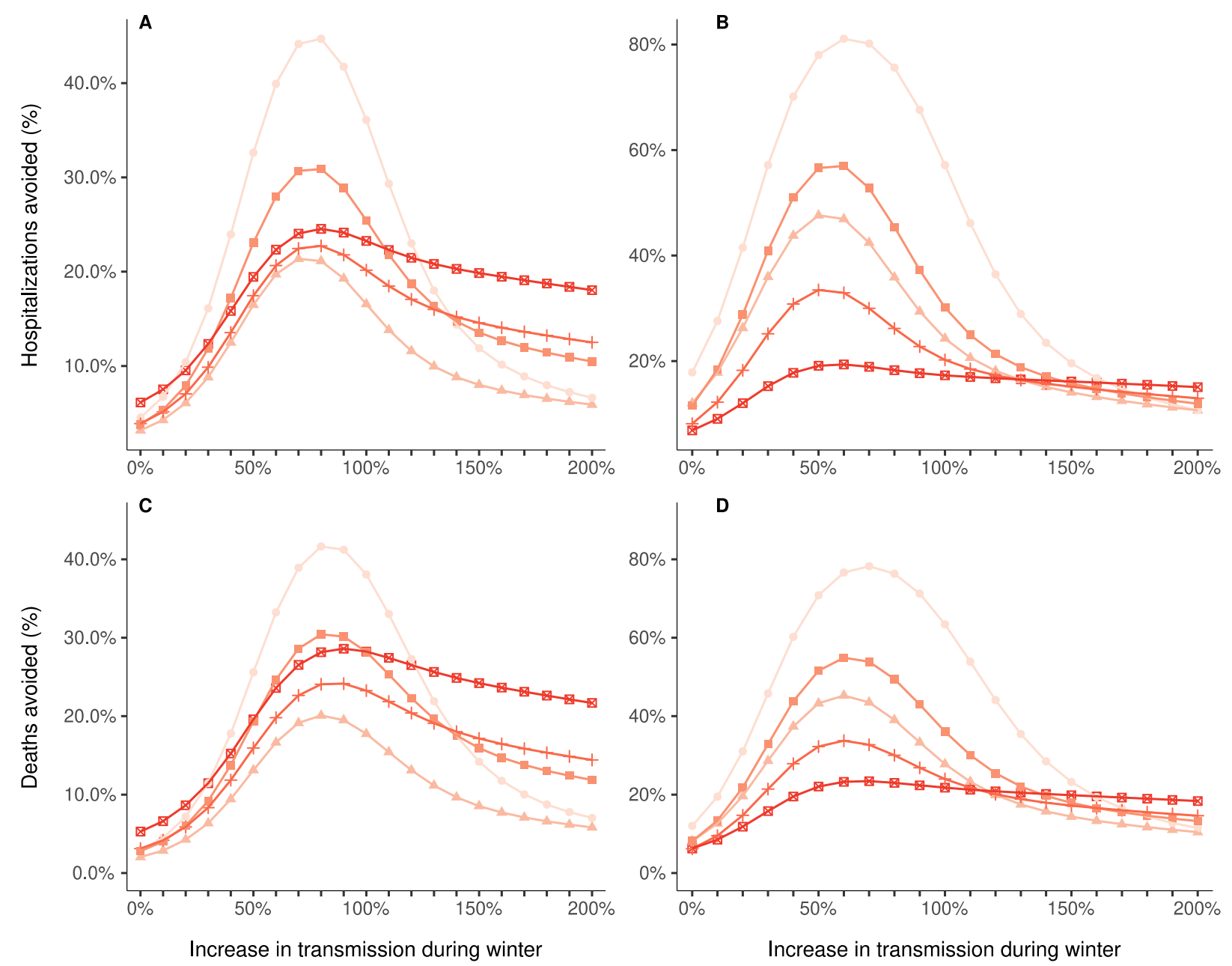

- Booster for 30-49 yo $\longrightarrow$ Booster for 50-64 yo $\rightarrow$ Booster for $30+$ yo + Booster for $50+$ yo $\rightarrow$ Booster for $65+$ yo

Figure 4. Proportion of hospitalizations (A,B) and deaths (C,D) avoided by 10 million booster shots compared to a baseline scenario of no booster shots, depending on the targeted age groups and the level of transmission during the winter, over the period from September 1st, 2021 to March 1st, 2022. In all strategies, primary vaccination is stopped on September 1st, 2021. The increase in transmission starts on September 20th, 2021, and is relative to the mean transmission levels during the summer $2021\left(R_{\text {eff }}=1.0\right)$. (A, C) Vaccine efficacy decreased only for people aged 65 years and older. (B, D) Vaccine efficacy decreased for all age groups. 


\section{Discussion}

Since a growing body of evidence shows that vaccine immunity to COVID-19 wanes with time ${ }^{12-}$ ${ }^{14}$ and decreases due to variants, ${ }^{4-6,12}$ several countries are implementing booster vaccination campaigns, however data is lacking regarding the effectiveness of such strategies. In a first analysis, we compared vaccination strategies that differed by the prioritization of the doses between primary and booster vaccination. Strategies prioritizing primary vaccination were systematically more effective than strategies prioritizing boosters, in both scenarios of decreased vaccine efficacy. Overall, the most beneficial strategy was to allocate all available doses to primary vaccinations and then use the remaining doses as boosters. This is expected if vaccine doses are preferentially administered to senior age classes because of their higher risk of hospitalization. However, the strategy remains valid also when primary vaccination is extended to the younger age classes. This confirms that, although the baseline overall vaccination coverage was already relatively high, increasing the number of vaccinated people has a greater impact on the epidemic dynamics than boosting the protection of elderly individuals already fully vaccinated. The results stratified by age groups also revealed the same patterns. Even people aged 65 years or older would benefit more from a strategy prioritizing new vaccinations (which would necessarily target younger age groups) than from a strategy prioritizing their own boosting. These results reinforce the fact that population immunity plays a greater role in decreasing the risks of vulnerable populations than their individual protection, thanks to the overall reduction of viral circulation in the population. However, this result could be affected by the application of recommendations or restrictions that mostly impact active adults (e.g. telework, closure of restaurants and bars or curfews).

We also analyzed the impact of booster strategies targeting different age groups, in a case where primary vaccination has reached a plateau. In the scenario in which vaccine efficacy is decreased for all age groups, the most effective strategy in order to reduce both hospitalizations and deaths was to boost the immunity of the 30-49 age group. In the scenario in which the vaccine efficacy is decreased only for the 65 years and older, the different boosting strategies had varying effectiveness depending on the level of virus transmissibility in the population, and the outcome considered (hospitalizations or deaths). These results suggest that, when deciding which populations to boost, two mechanisms must be considered: immunization of people with high risk of severe forms of the disease, but also immunization of people with high risk of transmission, which would reduce the viral circulation and therefore further protect the population at risk of severe consequences. Therefore, in the context of COVID-19, in which these two mechanisms involve different age groups, the optimal boosting strategy might be to target these two populations rather than only people above a certain age. Moreover, one must also consider the level of SARS-CoV-2 transmissibility in the population, which could be affected by seasonality, mitigation measures, or new variants.

These results must be interpreted with caution, as several assumptions and simplifications had to be made. Because of similarities between vaccines of the same type, we considered only two types of vaccines: mRNA vaccines and vector vaccines. This choice was made for simplification purposes, and because only one brand is dominant by vaccine type in France, with the Pfizer 
vaccine representing $88.6 \%$ of the vaccinations with mRNA vaccines, and the AstraZeneca vaccine $88.5 \%$ of the vaccinations with vector vaccines. ${ }^{9}$ Regarding the vaccine efficacies, we chose to consider distinct values depending on the predominant VOC on the French territory to account for the reduced efficacies against the Delta variant, ${ }^{5,6}$ and we considered two sets of vaccine efficacies against the Delta VOC, one in which the efficacies against Delta VOC are slightly lower than against Alpha VOC, and one in which the efficacies are greatly decreased for the protection against infection, according to recent studies. ${ }^{12-14}$ We considered a first scenario in which the efficacies are decreased only for people aged 65 years and older, and a second scenario, in which the efficacies are decreased for all age groups. However, we did not model the progressive decrease of vaccine efficacy over time, but rather changed the parameters at fixed time points. Also, data on the protection granted by a booster dose is still scarce, so we assumed that the efficacies obtained after a booster dose were equal to the values with the Alpha variant. A recent study from Israel ${ }^{23}$ and first results of clinical trials ${ }^{25}$ seem to confirm this assumption.

The vaccination strategies were designed arbitrarily considering that 200,000 doses could be administered daily, until a plateau of $90 \%$ coverage was reached. This choice of 200,000 daily doses was based on the observed number of doses used in France for first and second injections at the end of August $2021 .{ }^{9}$ The choice of $90 \%$ for the vaccination coverage plateau was based on the observed vaccination coverage reached in the elderly population in France. Although these parameters, together with the levels of virus transmissibility during winter, directly affect the results, sensitivity analyses showed that they only change the impact of the vaccination strategies in terms of absolute numbers, but that the ranking of the strategies remained unchanged (supplementary material). Moreover, the parameters associated with the natural history of SARS-CoV-2 infection used here are the ones of the original virus strain. Preliminary results from $\mathrm{Li}$ et al suggest that the average incubation time of the Delta variant could be as short as four days. ${ }^{26}$ Finally, our model also does not allow for a recovered individual to be infected a second time by the SARS-CoV-2 virus. Although cases of re-infection have been documented, ${ }^{27}$ not much is known about the risk of re-infection after having recovered from COVID-19. ${ }^{28}$ At this stage, it is not likely to impact the results, but it could be added later on when more data becomes available.

The model presented here is able to simulate age-specific, complex vaccination strategies that combine primary vaccinations and booster shots in various proportions, and at different rates. The analyses were performed in the context of metropolitan France; however, we expect that the conclusions drawn here would hold in many similar settings, that is countries where a portion of the population remains unvaccinated while the majority of the vaccinated individuals completed their vaccination four to five months before. While many countries have already announced plans for booster vaccine programs, others that struggle to achieve high coverage for the primary vaccinations have raised concerns regarding the equity in the access to vaccines. The WHO called into question the relevance of booster programs in the context of a pandemic where the majority of the world population has not received at least one dose. ${ }^{29}$ The recent emergence of the Omicron VOC ${ }^{30}$ illustrates how low vaccination coverage in large parts of the world might increase the risk that new, more potent variants emerge, which could in turn threaten even fully vaccinated countries. This work provides important insight in the matter of 
primary and booster vaccination, using the context of France as a case study, and can prove useful to inform these critical public health decisions.

Countries should continue pursuing primary vaccination in unvaccinated individuals to reduce hospitalizations and deaths. At the same time, with a rapid surge of cases in Europe due to the Delta variant and the threat of newly emerging variants, administering the booster doses to active adults would be overall more effective in mitigating the impact on the health system, under expected seasonal conditions of viral transmissibility.

\section{Acknowledgments}

The study was partially funded by the French national research agency (ANR) through the project SPHINX-17-CE36-0008-0. The authors thank Daniel Levy-Bruhl and Simon Cauchemez for fruitful discussions regarding this work.

\section{Authors' contributions}

CRM, JR, and PC designed and planned the study. PC developed the initial mathematical model. CRM, JR, and PC further developed the model for this study. CRM and JR accessed official data on COVID-19 epidemiology and vaccination in France. CRM and JR performed the analyses. CRM, JR, and PC audited the code. CRM wrote the first draft of the manuscript. JR, PC, and VC critically commented on the primary and sensitivity analyses, and suggested amendments. All authors contributed to revisions of the manuscript. The corresponding author made the decision to submit the manuscript for publication

\section{Conflicts of interest}

PC has received consulting fees from Sanofi-Pasteur for projects unrelated to COVID-19 and unrelated to this project. All other authors declare no potential conflicts of interest. 


\section{References}

1 Polack FP, Thomas SJ, Kitchin N, et al. Safety and efficacy of the BNT162b2 mRNA Covid-19 vaccine. N Engl J Med 2020.

2 Abu-Raddad LJ, Chemaitelly H, Butt AA. Effectiveness of the BNT162b2 Covid-19 Vaccine against the B.1.1.7 and B.1.351 Variants. N Engl J Med 2021. DOI:10.1056/NEJMc2104974.

3 Bernal JL, Andrews N, Gower C, et al. Effectiveness of the Pfizer-BioNTech and OxfordAstraZeneca vaccines on covid-19 related symptoms, hospital admissions, and mortality in older adults in England: test negative case-control study. bmj2021; 373.

4 Sheikh A, McMenamin J, Taylor B, Robertson C. SARS-CoV-2 Delta VOC in Scotland: demographics, risk of hospital admission, and vaccine effectiveness. Lancet 2021; 397: 24612.

5 Lopez Bernal J, Andrews N, Gower C, et al. Effectiveness of Covid-19 Vaccines against the B.1.617.2 (Delta) Variant. N Engl J Med 2021; 385: 585-94.

6 Stowe J, Andrews N, Gower C, et al. Effectiveness of COVID-19 vaccines against hospital admission with the Delta (B.1.617.2) variant. khub 2021; : 3.

7 Haas EJ, Angulo FJ, McLaughlin JM, et al. Impact and effectiveness of mRNA BNT162b2 vaccine against SARS-CoV-2 infections and COVID-19 cases, hospitalisations, and deaths following a nationwide vaccination campaign in Israel: an observational study using national surveillance data. Lancet 2021; 397: 1819-29.

8 Agence nationale de sécurité du médicament. COVID-19 - Vaccins autorisés. 2021. https://ansm.sante.fr/dossiers-thematiques/covid-19-vaccins/covid-19-vaccins-autorises.

9 Santé Publique France. Données relatives aux personnes vaccinées contre la Covid-19. 2021. https://www.data.gouv.fr/fr/datasets/donnees-relatives-aux-personnes-vaccinees-contre-lacovid-19-1/.

10 Santé Publique France. COVID-19: point épidémiologique du 11 mars 2021. 2021. https://www.santepubliquefrance.fr/maladies-et-traumatismes/maladies-et-infectionsrespiratoires/infection-a-coronavirus/documents/bulletin-national/covid-19-pointepidemiologique-du-11-mars-2021.

11 Santé Publique France. COVID-19: point épidémiologique du 16 juillet 2021. 2021. https://www.santepubliquefrance.fr/maladies-et-traumatismes/maladies-et-infectionsrespiratoires/infection-a-coronavirus/documents/bulletin-national/covid-19-pointepidemiologique-du-16-juillet-2021.

12 Tartof SY, Slezak JM, Fischer $\mathrm{H}$, et al. Effectiveness of mRNA BNT162b2 COVID-19 vaccine up to 6 months in a large integrated health system in the USA: a retrospective cohort study. The Lancet 2021; 398: 1407-16.

13 Chemaitelly H, Tang P, Hasan MR, et al. Waning of BNT162b2 Vaccine Protection against SARS-CoV-2 Infection in Qatar. N Engl J Med 2021; published online Oct 6.

DOI:10.1056/NEJMoa2114114.

14 Goldberg Y, Mandel M, Bar-On YM, et al. Waning Immunity after the BNT162b2 Vaccine in Israel. N Engl J Med 2021; : NEJMoa2114228.

15 Haute Autorité de Santé. Avis n²021.0061/AC/SEESP du 23 août 2021 du collège de la HAS relatif à la définition des populations à cibler par la campagne de rappel vaccinal chez les 
medRxiv preprint doi: https://doi.org/10.1101/2021.12.01.21267122; this version posted December 2, 2021. The copyright holder for this preprint (which was not certified by peer review) is the author/funder, who has granted medRxiv a license to display the preprint in perpetuity. It is made available under a CC-BY-NC-ND 4.0 International license .

personnes ayant eu une primovaccination complète contre la Covid-19. 2021 https://www.has-sante.fr/jcms/p_3283044/fr/avis-n-2021-0061/ac/seesp-du-23-aout-2021du-college-de-la-has-relatif-a-la-definition-des-populations-a-cibler-par-la-campagne-derappel-vaccinal-chez-les-personnes-ayant-eu-une-primovaccination-complete-contre-lacovid-19.

16 [preprint] Roux J, Massonnaud C, Colizza V, Cauchemez S, Crépey P. Impact of national and regional lockdowns on COVID-19 epidemic waves: Application to the 2020 spring wave in France. medRxiv. 2021. DOI:10.1101/2021.04.21.21255876.

17 Kiem CT, Massonnaud CR, Levy-Bruhl D, et al. A modelling study investigating short and medium-term challenges for COVID-19 vaccination: From prioritisation to the relaxation of measures. EClinicalMedicine 2021; 38: 101001.

18 Davies N, Klepac P, Liu Y, et al. Age-dependent effects in the transmission and control of COVID-19 epidemics. Nat Med 2020; 26: 1205-11.

19 Prem K, Cook AR, Jit M. Projecting social contact matrices in 152 countries using contact surveys and demographic data. PLOS Comput Biol2017; 13: 1-21.

20 Bager, P. and Wohlfahrt, J. and Rasmussen, M. and Albertsen, M and Grove Krause, T. Hospitalisation associated with SARS-CoV-2 delta variant in Denmark. Lancet Infect Dis 2021; published online Sept.

21 Collin A, Hejblum BP, Vignals C, et al. Using Population Based Kalman Estimator to Model COVID-19 Epidemic in France: Estimating the Effects of Non-Pharmaceutical Interventions on the Dynamics of Epidemic. 2021.

22 Carrat F, de Lamballerie X, Rahib D, et al. Antibody status and cumulative incidence of SARSCoV-2 infection among adults in three regions of France following the first lockdown and associated risk factors: a multicohort study. Int J Epidemiol 2021; 50: 1458-72.

23 Barda N, Dagan N, Cohen C, et al. Effectiveness of a third dose of the BNT162b2 mRNA COVID19 vaccine for preventing severe outcomes in Israel: an observational study. The Lancet 2021; 0. DOI:10.1016/S0140-6736(21)02249-2.

24 Haute Autorité de Santé. Stratégie de vaccination contre le Sars-Cov-2 - Recommandations préliminaires sur la stratégie de priorisation des populations à vacciner. 2021 https://www.has-sante.fr/jcms/p_3221338/fr/strategie-de-vaccination-contre-le-sars-cov-2recommandations-preliminaires-sur-la-strategie-de-priorisation-des-populations-avacciner.

25 Pfizer and BioNTech Announce Phase 3 Trial Data Showing High Efficacy of a Booster Dose of Their COVID-19 Vaccine | Pfizer. https://www.pfizer.com/news/press-release/press-releasedetail/pfizer-and-biontech-announce-phase-3-trial-data-showing (accessed Nov 12, 2021).

$26 \mathrm{Li} \mathrm{B}$, Deng A, Li K, et al. Viral infection and transmission in a large well-traced outbreak caused by the Delta SARS-CoV-2 variant. MedRxiv 2021.

27 Iwasaki A. What reinfections mean for COVID-19. Lancet Infect Dis 2021; 21: 3-5.

28 Boyton RJ, Altmann DM. Risk of SARS-CoV-2 reinfection after natural infection. The Lancet 2021; 397: 1161-3.

29 World Health Organization. Interim statement on COVID-19 vaccine booster doses. 2021 \{https://www.who.int/news/item/10-08-2021-interim-statement-on-covid-19-vaccinebooster-doses\}.

30 WHO. Classification of Omicron (B.1.1.529): SARS-CoV-2 Variant of Concern. 2021; published 
medRxiv preprint doi: https://doi.org/10.1101/2021.12.01.21267122; this version posted December 2, 2021. The copyright holder for this preprint (which was not certified by peer review) is the author/funder, who has granted medRxiv a license to display the preprint in perpetuity.

\author{
It is made available under a CC-BY-NC-ND 4.0 International license .
}

online Nov 26. https://www.who.int/news/item/26-11-2021-classification-of-omicron(b.1.1.529)-sars-cov-2-variant-of-concern. 